\title{
Concept and realization of 2D and 1D detector readout systems with new adjustment and calibration features
}

\author{
P. Rasmussen ${ }^{1, *}$, J. Kohlbrecher ${ }^{2}$, J. Egger ${ }^{1}$, J. Mesot $^{3}$, M. Schild $^{2}$, R. Baetscher ${ }^{1}$, E. Berruyer ${ }^{4}$, N. Schlumpf ${ }^{1}$ \\ ${ }^{1}$ Laboratory for Particle Physics, Paul Scherrer Institute, 5232 Villigen-PSI, Switzerland \\ ${ }^{2}$ Spallation Neutron Source (SINQ), Paul Scherrer Institute, 5232 Villigen-PSI, Switzerland \\ ${ }^{3}$ Laboratory for Neutron Scattering, ETH Zuerich \& PSI Villigen, 5232 Villigen-PSI, Switzerland \\ ${ }^{4}$ Compagnie pour l' Etude et la Realisation de Combustibles Atomiques (CERCA), 26104 Romans-sur-Isere, France
}

Received: 17 July 2001/Accepted: 24 October 2001 - (C) Springer-Verlag 2002

\begin{abstract}
We present the concept and report on the realization of the new 2D readout system for the CERCA $128 \times$ $128-7.5 \mathrm{~mm}^{2}$-element neutron detection chamber installed on the SINQ-SANS instrument. The adjustment and calibration of the detector response is performed iteratively under software control. This new development includes an additional time of flight (TOF) option. Based on this successful design, we are now developing a 1D TOF readout system for the SINQ-POLDI detection unit.
\end{abstract}

PACS: 07.05.Hd; 07.50.Qx; 84.30.-r

We decided to develop a new electronic readout system for the CERCA $128 \times 128-7.5 \mathrm{~mm}^{2}$-element $x / y$ area-sensitive detector installed on the SINQ-SANS instrument [1] $]^{1}$ after several unsuccessful attempts to bring the detector system up to the specified performance [2].

\section{Concept}

The existing detector layout called for a "geographical" splitting of the functionality into distinct building blocks (modules).

\subsection{Channel boards}

Each of the 256 wires is read out by a charge/voltage converter (CVC) followed by a pole zero cancellation (PZC) stage and a shaping-amplifier unit. An initial "hard-wired" adjustment of the sensitivity of the analog part of the channel electronics to $\pm 1 \%$ is required. Further, the temperature dependence of the sensitivity and the equivalent noise charge (ENC) at the input of this subcircuit had to be minimized. The A/D conversion stage provides four individually remote adjustable thresholds in order to be able to optimize the "homogeneity of the detector response" iteratively by the host computer.

${ }^{*}$ Corresponding author. (E-mail: peter.rasmussen@psi.ch)

${ }^{1}$ http://sinq.web.psi.ch/sinq/instruments.html

\subsection{Front-end box}

Due to the existing distribution of the connectors on the back of the multi-wire proportional chamber (MWPC), each front-end box houses 32 channel boards on two motherboards with the corresponding front-end logic: each "triple-wire event" is attributed to one out of three wires by a combination of low-level discrimination - "center-of-charge" estimation - and pile-up rejection logic. The 8 front-end boxes transmit their prefiltered data items to a central coordination unit: the data-gathering module.

\subsection{Data-gathering module}

This unit is centered on the rear of the detector body with approximately equal-length wiring to the front-end boxes. The event "time stamps" are derived from a stop-watch clock that is able to be externally synchronized. They allow for the recognition of the $x / y$ coincident events (position information) and the running of the SANS instrument in the stroboscopic mode (time information). The "good events" (position and time information) found together with the system-status information are transmitted to the PSI "Histo-Memory" unit [3] by special implementation of the PSI "Multidetector Interface". The data-gathering module mainly consists of a micro-controller unit (MCU) with some field-programmable gate arrays (FPGAs) performing the required tasks. It is fully programmable through an optical-fiber link.

\subsection{Data-transmission unit}

The data stream from the detector is transmitted continuously from the data-gathering module to the external PSI Histo-Memory through a second optical link providing a datatransmission capability of $100 \mathrm{MBit} / \mathrm{s}$. By including the detector-status information in the main data stream every $100 \mathrm{~ms}$, important instrument parameters and the data-link integrity are continuously checked by the Histo-Memory processor unit. 


\section{Analog subcircuits}

Considering the given $70-80-\mathrm{pF}$ capacitances of the 256 cathode wires, the new channel boards were designed to provide under such working conditions an ENC at the input of

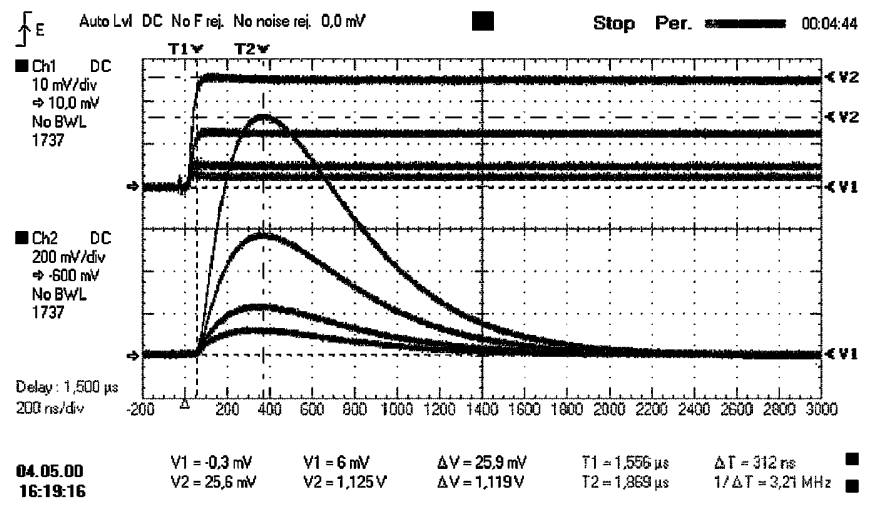

Fig. 1. Scope snapshot (persistence mode) showing the channel-board performance (noise, sensitivity): Ch1: 3.46, 6.86, 17.3 and $34.7 \mathrm{fC}$ are delivered to the gate of the JFET at the input of the CVC circuit. $\mathrm{Ch} 2$ : the corresponding shaping-amplifier output signals

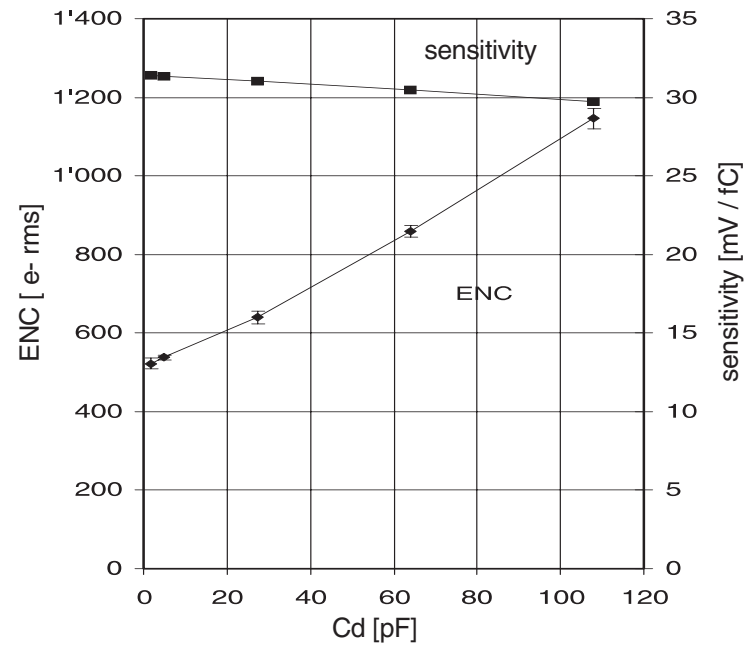

Fig. 2. Analog subcircuits: measured ENC and sensitivity slopes as a function of detector capacitances up to $100 \mathrm{pF}$

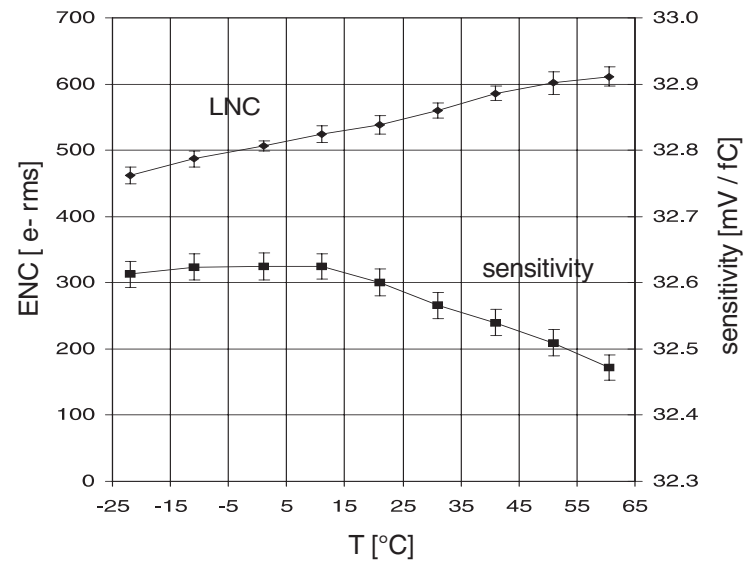

Fig. 3. Analog subcircuits: measured ENC and sensitivity slopes due to temperature variations

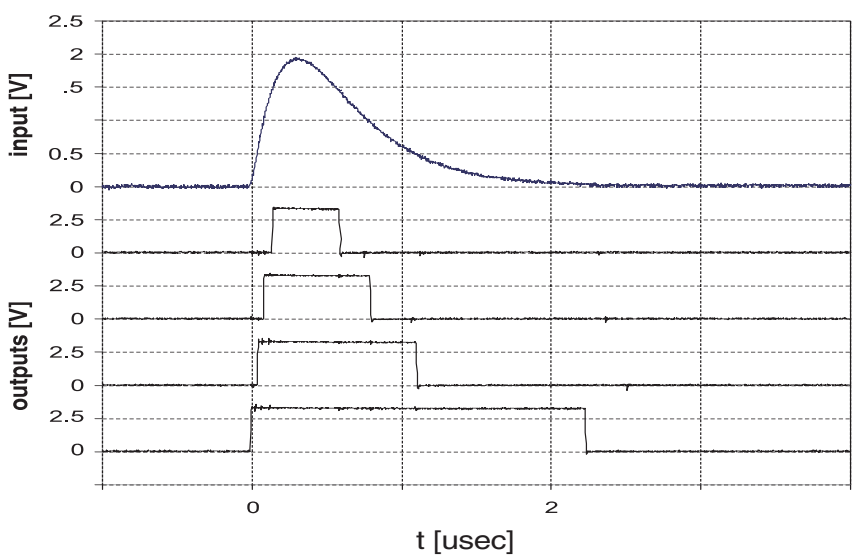

Fig. 4. A/D-conversion-stage response to an analog input signal crossing all four comparator threshold levels

$\leq 1000 e_{\text {rms }}^{-}$. The shaping amplifier could be configured to obtain a peaking time of about $300 \mathrm{~ns}$, and the sensitivity was set to $\simeq 30 \mathrm{mV} / \mathrm{fC}$, allowing for reasonable gas gains in the detection chamber of about 25 (Fig. 1). Measured typical noise and sensitivity slopes are presented in Fig. 2. The temperature dependence of the ENC and the sensitivity were also analyzed (Fig. 3). In the relevant temperature range $\left(20-60^{\circ} \mathrm{C}\right)$ an accuracy of $\leq 100 \mathrm{ppm} / \mathrm{K}$ was obtained for the temperature sensitivity of the channel boards. The signal at the output of the analog circuit is presented to the input of a 4-fold comparator stage with remotely programmable thresholds to be converted to a digital "4-bit value" (Fig. 4) for further treatment in the pile-up rejection and center-of-charge estimation logic.

\section{Front-end logic and event grabber}

If a wire event is detected, i.e. the lowest discriminator level is activated, a programmable time window of up to $1.6 \mu \mathrm{s}$ is started. During this time a center-of-charge estimation is performed through the presentation of the 4-bit values (Fig. 4) from a maximum of three adjacent wires to a preprogrammed lookup table in the FPGAs. If this logic detects a second peak (i.e. a 4-bit value increases anew) before all the involved comparators have released their corresponding outputs, this pile-up event is rejected. In the case where more than three adjacent wires detect an amount of charge above the lowest threshold level, an error message is generated. Up to this stage the $128 X$ and the $128 Y$ wires are treated as individual $1 \mathrm{D}$ detectors.

The event logic unit in the data-gathering module analyzes the $X$ and $Y$ data entities by applying a programmable coincidence window to the presented information. Recognized good events are finally characterized by the attributed $X$ - and $Y$ wire numbers and a time stamp (possibly related to an external synchronization signal) with a selectable resolution down to $100 \mathrm{~ns}$.

Further, we implemented a very useful, programmable analog pulser system with remotely adjustable amplitudes, frequency and time delays. This allows for quick tests and functionality checks on the entire electronic path, starting from the wire (charge) inputs up to the Histogramming Memory in the VME crate. At start-up the default system configuration is 
loaded into the FPGAs by the MCU. This MCU also allows for interactive operation of the detection system, reprogramming of the wire thresholds, modification of the FPGA settings (overwriting the configuration information) and programming of the test pulser system.

\section{Detector-response calibration}

The SANS detector was displaced in 3-mm steps in front of a narrow $(1.5 \mathrm{~mm})$ neutron beam, and the pulse-height spectra from one single wire were measured in order to obtain reasonable starting values for the iterative adjustment procedure of the individual wire-event detection thresholds (Fig. 5). The threshold leading to the Gaussian-shaped curve (max. $\simeq 0.6)$, where a negligible number of events outside $\pm 11 \mathrm{~mm}$ are accumulated, was selected (about $\pm 1.5 \times$ the wire distance).

In the response calibration algorithm the individual wirecount-rate deviations from $\frac{1}{128}$ th of the overall detector count rate were damped by a factor of 0.4 in order to obtain smooth convergence of the iteration process. After only five iterations a response homogeneity of about $1 \%$ was achieved.

\section{First results and outlook}

First tests confirmed that this system is able to cope with neutron rates up to 100000 events/s. The estimated dead time per event (1-2 $\mu \mathrm{s}$ is mainly due to the analog signal treatment time together with the event threshold values selected. During a service shutdown of the SINQ source an overall detector background count rate of $1.05(0.02)$ counts/s was measured.

To confirm the convergence of our response calibration procedure, a diffraction pattern of dissolved polystyrene was recorded (Fig. 6). A further example of the excellent system performance is presented in Fig. 7: a diffraction pattern of the vortex lattice from a high-temperature superconductor in a magnetic field [4]. This data set was obtained by subtract-

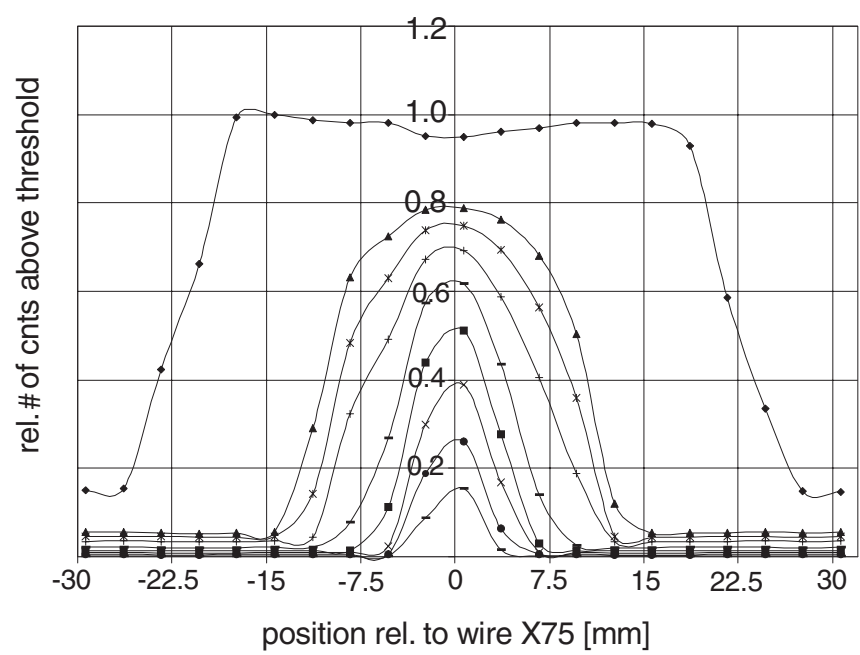

Fig. 5. SANS detector scan in front of a narrow neutron beam (1.5-mm wide, $\| X$; bias voltage on anode grid: $2.6 \mathrm{kV}$ ) with wire-event detection threshold variations from $110 \mathrm{mV}$ up to $2.8 \mathrm{~V}$ (lowest level on wire $X 75$ )

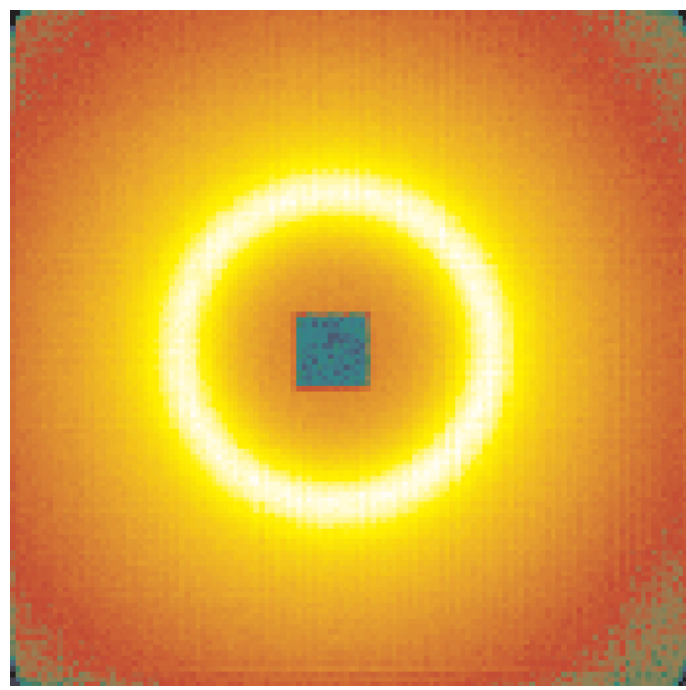

Fig. 6. Diffraction pattern (raw data) of dissolved polystyrene. The square in the center is the image of the neutron absorber positioned in front of the detection chamber

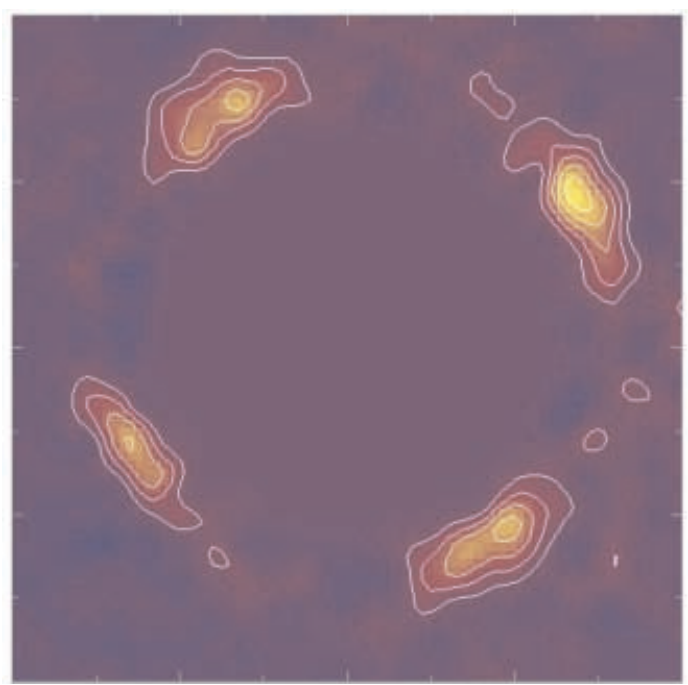

Fig. 7. Diffraction pattern of the vortex lattice in a high-temperature superconductor in a magnetic field of $0.8 \mathrm{~T}$ applied along the $c$-direction

ing two raw diffraction images with a magnetic field either switched on or off. This kind of experiment relies on the excellent "long-term" stability of the measuring system. Tests showed that differences calculated from two data sets accumulated under identical conditions at time intervals up to $24 \mathrm{~h}$ apart $(\Delta T \leq 8 \mathrm{~K})$ levelled off well below $1 \%$.

Based on the successful development for the CERCA 2D detector, we are adapting this design now to the in-house-built POLDI detection chamber, a 400-wire 1D detection system with a remote selectable time resolution down to $100 \mathrm{~ns}$ in time-of-flight mode.

\section{References}

1. J. Kohlbrecher et al.: J. Appl. Cryst. 33, 804 (2000)

2. PSI internal documents

3. U. Greuter et al.: Appl. Phys. A 74, S142 (2002)

4. J. Mesot et al.: to be published 